\title{
HYPOTHETICAL DARK MATTER/AXION ROCKETS: DARK MATTER IN TERMS OF SPACE PHYSICS PROPULSION
}

\author{
A. BECKWITH \\ American Institute of Beam Energy Propulsion \\ P.O. Box 1907 \\ Madison, Alabama, 35758 \\ 650-322-6768 \\ E-mail:abeckwith@uh.edu
}

\begin{abstract}
Current proposed photon rocket designs include the Nuclear Photonic Rocket and the Antimatter Photonic Rocket (proposed by Eugen Sanger in the 1950s, as reported by Ref. 1). This paper examines the feasibility of improving the thrust of photon-driven ramjet propulsion by using DM rocket propulsion. The open question is: would a heavy WIMP, if converted to photons, upgrade the power (thrust) of a photon rocket drive, to make interstellar travel a feasible proposition?
\end{abstract}

Keywords: Dark Matter, Photon Rocket, Axions

PACS: 07.87.+v, 95.85.Ry, 95.35.+d

\section{Which DM candidates are viable?}

Reference 2 provides a ten-point test a new particle has to pass to be considered a viable DM candidate: "(I) Does it match the appropriate relic density? (II) Is it cold? (III) Is it neutral? (IV) Is it consistent with BBN? (V) Does it leave stellar evolution unchanged? (VI) Is it compatible with constraints on self-interactions? (VII) Is it consistent with direct DM searches? (VIII) Is it compatible with gamma-ray constraints? (IX) Is it compatible with other astrophysical bounds? (X) Can it be probed experimentally?" It so happens that WIMPs meet all the above tests. Reference 3 gave a mass value of between 100-300 GeV for WIMPs. A second theory involving undetected particles is that some dark matter is made of hypothetical subatomic particles called "axions." Thus, the difference between the two theories (WIMPs or axions) is that dark matter is either made of a large number of light particles (axions) or a smaller number of heavier particles. 
A way to link the two states of DM is to note, as Ref. 4 notes, axions were the whole of $\mathrm{DM}$, that there would be $m_{\text {axion }} \approx 10^{-5} \mathrm{eV}$ for a total DM mass $M \approx 10^{12} \mathrm{GeV}$. But if there were WIMPs and axions together, $\left.\left.m_{\text {axion }} \approx 10^{-5} \mathrm{eV}\right|_{\text {no-WIMPs }} \gg m_{a}\right|_{\text {WIMPs-exist }}$. For most theoreticians, neutralinos are the preferred SUSY particle of choice for DM. However, in what has startling implications, Refs. 5 and 6 using Mesissner and Nicholai's parameter space arguments obtained WIMP DM masses of between $300 \mathrm{GeV}$ to $400 \mathrm{GeV}$ as an upper range to WIMP masses. What needs to be obtained, is analytical work to fathom an interrelationship between axions and WIMPs as far as DM. Possibly by Bayesian statistical methods for comparing the relative fit between WIMP and axion models, i.e., effective Lagrangian methods as explored by Ref. 7. Axions have been considered as a power source to be scooped up in space because of their estimated thick density in space, and DM candidates for masses considerably above the axion values have been brought up as a way to increase thrust / power for more efficient propulsion. Reference 8 writes that a typical Daedalus's star ship designed for six light years of travel requires 1.7 million metric tons of fuel, which is unrealistic, and Frisbee states that the photon rocket has a travel time of 42 million years to accelerate to one tenth the speed of light.

\section{First Principles of an Axion/DM Ramjet}

According to Ref. 9 in discussions of the applications of their description of the CAST experiment, axions can be changed by the Primakoff effect into photons, which could theoretically be used as a source of thrust. ${ }^{9,10}$ The power density available from axions depends on their mass, the density of axions in space, and the velocity of the vehicle. At $10^{-5} \mathrm{eV}$, with a velocity of .001c, assuming 200,000 per cc axions in intergalactic space (an axion has a mass $1 / 400,000,000$ th of the mass of an electron, so there should be 200,000 per cc in intergalactic space, according to Ref. 11).

$$
\text { Power }=3 \mathrm{watts} / \mathrm{cm}^{2} \times[v / c] /\left(1-[v / c]^{2}\right) .
$$

The author used a velocity of .001c, assuming a ramjet is used for inserting axions into a chamber from outer space. At $.999 \mathrm{c}$, the power is nearly 1500 watts $/ \mathrm{cm}^{2}$, which appears to be respectable. These calculations assume a density of half a trillion axions per cubic centimeter the vicinity of Earth, more per cc near the galactic center, and only is also assumed that an axion traveling at .1c, hitting a $1 \mathrm{~cm}$-squared region of space, undergoes an $10^{-5}$ 
electron volt value to $10^{-3} \mathrm{eV}$ value for the axion mass. This axion mass would then be directly converted into energy, and that there are roughly up to half a trillion axions per cubic centimeter. So how can one obtain a power value of 1500 or so watts per square centimeter at nearly .1c? What is being looked for is how to have a far greater energy power equivalent to 1500 Watts per square centimeter for far slower travel. The reason being that the greater the power output at lower speed of light values, the faster a space craft would be able to accelerate to that speed. Our Dark Matter candidates (WIMPs), instead of being $10^{-3} \mathrm{eV}$ are, instead, 100 to 400 $\mathrm{GeV}$. So a more efficient way is required to reach a power ratio of 1500 or so watts per square centimeter for a rocket. The problem of momentum kick is as follows. As implied by Ref. 12, "Every axion produces a momentum kick of

$$
\Delta p=m c \times \gamma \cdot(1-\beta)
$$

where $m$ is the axion rest mass." Special relativity suggests that a lowpower-output space craft would take quite a long time to accelerate to almost the speed of light. A viable DM rocket would allow a rocket to have far more power, permitting a more rapid acceleration to at least ten percent of the speed of light in a reasonable time period.

\section{And why this is all important? Facing some serious problems in contemporary cosmology}

At the EXTREMA of any function: $d x / d t=0$. But for Big-Bang, $d S / d t=$ $\infty$ at $S=0$. And then why would one care about entropy in the first place. It so happens as reported by Ref. 6 as adapted from Refs. 13 and 14. The fact is that the non-SUSY Lagrangian offered by Ref. 5 may not only give the correct mass value for a useful interstellar propulsion candidate, but also tie in with entropy production formalism which avoids $d S / d t=\infty$ at $S=0$ seen in present cosmology models. It is then appropriate to consider practical applications of Reference 14's quantum 'infinite' statistics. Reference 14 outlines how to get $S \approx N$. Begin with a partition function

$$
Z_{N} \sim\left(\frac{1}{N !}\right) \cdot\left(\frac{V}{\lambda^{3}}\right)^{N} .
$$

This, according to $\mathrm{Ng}$, leads to an entropy of the limiting value of 


$$
S \approx N \cdot\left(\log \left[V / N \lambda^{3}\right]+5 / 2\right) .
$$

But $V \approx R_{H}^{3} \approx \lambda^{3}$, so unless $N$ in Eq. (4) above is about $1, S$ (entropy) would be $<0$, which is a contradiction. Now this is where $\mathrm{Ng}$ introduces removing the $N$ ! term in Eq. (3) above, i.e., inside the Log expression we remove the expression of $N$ in Eq. (4) above. This is a way to obtain what $\mathrm{Ng}$ refers to as Quantum Boltzmann statistics, so then we obtain for sufficiently large $N$, where $N$ is a numerica DM density referred to as $<n>$.

$$
S \approx N
$$

\section{Conclusion}

One can state that near-light speeds, the available axion power would be about 3 watts $/ \mathrm{cm}^{2}$ times $\beta \times \gamma^{2}$, where $\beta=(v / c)$ is the velocity relative to light, and $\gamma^{2}=1 /\left[1-\beta^{2}\right]$ is the square of the relativistic mass-increase factor. At a velocity of $99.9 \% \mathrm{c}$, the available power from axions would be 1500 watts $/ \mathrm{cm}^{2}$, enough power for a modest energy-efficient space drive (the faster it goes, the more such power becomes available). In principle, a photon rocket may be improved upon, using DM/axion destruction via intense E \& B fields. In IDM 2008, a mass range for DM up to about $400 \mathrm{GeV}$, per particle was predicted, adding credibility to a counting algorithm based on Eq. (5). ${ }^{6}$ I.e., entropy would then be added due to DM particles which would be produced within the CMBR region of space. I.e., the wave length of DM would be well within the region of space before 380 thousand years after the Big Bang. What is needed, to make a linkage between axions and WIMP DM more understood would be application of Bayesian statistics, as Ref. 7 wrote about. An experimental program of DM applications to space flight may enable investigations into this issue, allow for understanding the genesis of entropy in pre CMBR space, and lead to engineering studies in order to make interstellar travel a possibility.

\section{References}

1. A. V. Gulevich, E. A. Ivanov, O. F. Kukharchuk, V. Y. Poupko and A. V. Zrodnikov, STAIF 552, 957 (2001).

2. M. Taoso, G. Bertone and A. Masiero, Journal of Cosmology and Astrophysics JCAP03(2008)022(March 2008). 
3. H. Muramaya, Physics beyond the standard model and dark matter, in Lectures at Les Houches Summer School, Session 86, Particle Physics and Cosmology: the Fabric of Spacetime, 31 July-25 August 2006.

4. S. Weinberg, Cosmology (Oxford University Press, New York, 2008).

5. K. A. Meissner and H. Nicolai(September 2008), arXiv:0803.2814v3 [hep-th].

6. A. W. Beckwith(24-27 February 2009), arXiv:0810.1493 [physics.gen-ph].

7. F. Feroz, B. C. Allanach, M. Hobson, S. S. AbdusSalam, R. Trotta and A. M. Weber(July 2008), arXiv:0807.4512v1 [hep-ph].

8. R. H. Frisbee, Limits of intestellar flight technology, in Frontiers Propulsion Science, eds. M. Millis and E. Davis, Progress in Atronautics and Aeronautics, Vol. 227 (AIAA, 2009) pp. 31-126.

9. J. I. Collar, D. Miller, J. Rasmussen and J. Vieira (2006).

10. R. Bernabei, P. Belli, R. Cerulli, F. Montecchia, F. Nozzoli, A. Incicchitti, D. Prosperi, C. J. Dai, H. L. He, H. H. Kuang, J. M. Ma and S. Scopel, Physics Letters B 515, 6(August 2001).

11. B. Lakic, Search for solar axions with the cast experiment, in Proceedings of IDM 2008, (Stockholm, Sweden, 2008).

12. P. Sikivie, Physical Review Letters 51, 1415 (1983).

13. Y. J. Ng, Quantum foam and dark energy, in International workshop on the Dark Side of the Universe, 2008. http://ctp.bue.edu.eg/workshops/Talks/ Monday/QuntumFoamAndDarkEnergy.pdf.

14. Y. J. Ng, Entropy 10, 441 (2008).

15. A. W. Beckwith(January 2009), arXiv:0809.1454v3 [physics.gen-ph].

16. K. A. Meissner. personal communication, Bad Honnef meeting on "Quantum gravity, new directions and perspectives".

17. P. Giromini, F. Happacher, M. J. Kim, M. Kruse, K. Pitts, F. Ptohos and S. Torre(October 2008), arXiv:0810.5730v1 [hep-ph]. 